\title{
A Framework to Support E-Commerce Development for People with Visual Impairment
}

\author{
Hulya Francis, Dhiya Al-Jumeily, \\ Applied Computing Research Group, \\ Liverpool John Moores University, \\ Byrom Street, L3 3AF, Liverpool, UK \\ h.francis@1jmu.ac.uk
}

\author{
Tom Oliver Lund \\ Liverpool John Moores University, \\ Byrom Street, L3 3AF, Liverpool, UK
}

\begin{abstract}
World Wide Web Consortium provides software developers with guidelines for designing accessible, cross browser compatible websites. Currently however, there are no guidelines in this area specific to the features of an e-commerce website.

This paper explores the current usability issues relevant to users with visual impairment and further proposes a framework that seeks to ensure the site is suitable for visually impaired users. This paper includes data analysis which compares current issues in web technologies suitable for e-commerce and proposes system adaptations which can be conducted to portray the improvements in overall user experience. To this extent, several validation tools and testing techniques have been used to identify the usability issues that visually impaired users currently face when shopping online.
\end{abstract}

Keywords-compatible; funtional prototype; visual impairments; $e$ commerce; validation tools.

\section{INTRODUCTION}

The primary objective of the study is to create a narrative set of guidelines to assist users in designing and developing an e-commerce interface. An investigative analysis of the current major E-businesses, for example; Amazon and Ebay will be conducted in order to explore various website usability issues. Several validation tools and techniques as provided by the Worldwide Web Consortium (W3C) and other websites will be used in order to understand the usability issues.

Until recently, business transactions conducted by the use of Electronic Data Interchange (EDI) over value-added networks (VAN) whereby companies would transfer information of similar format in a computer-to-computer exchange using two translator machines. However, according to Zwass (1996), "traditional e-commerce, conducted with the use of information technologies centreing on electronic data interchange (EDI) over proprietary value-added networks, is rapidly moving to the Internet." [1]. Since 2003, e-commerce grew rapidly, quicker than it had initially in the mid 1990's and this upturn was widely regarded as the 2 nd wave of ecommerce [2]. Webloyalty, an e-commerce specialist business, have produced figures that state; "online retail is growing six times faster than high street sales and is predicted to exceed $£ 37$ billion by 2014" [3]. An e-commerce websites main goal is to make a good first impression on its target audience. If an e-commerce website does not make a good first impression, potentially clients will be lost. E-commerce has revolutionized the way business is conducted and the way in which consumers purchase products and services.

\section{E-COMMERCE AUDIT}

In this section an analysis of various e-commerce websites have been tested for conformity to current UK accessibility legislation and standards with the aim of identifying the current issues. The major e-commerce website "amazon.co.uk" has been used for this part of the research. It is not always possible to test each and every page of an e-commerce website as many e-commerce websites are vast and include thousands of pages of products, services and information. Therefore, it is necessary that a few selected pages are targeted to represent the entire website. For the purpose of this paper the main functional pages of the e-commerce websites will be tested, including the home page, search related pages, product pages and checkout.

The Disability Discrimination Act (DDA, 1995) has been replaced by the Equality Act ( $1^{\text {st }}$ October 2010). However, the Disability Equality Duty in the DDA continues to apply in the current political system in the UK. "The Disability Discrimination Act (DDA), (1995) requires all high street businesses to provide disabled users with provisional access unless it is deemed impractical. The law states that it is unlawful to treat a disabled person less favourably than an able bodied person for any reason in relation to their disability. Service providers have to make reasonable adjustments in order to facilitate a disabled person. Therefore, physical adaptations to real world properties to aid accessibility should be replicated online. An online equivalent to access ramps and automatic doors to buildings would be necessary to meet the requirements of all users without discriminating against disabled users" [4].

\section{A. Methodologies}

In order to obtain information on the usability issues of the current major e-commerce websites, several testing and evaluation tools unique to accessibility will be used, providing a report on the accessibility issues discovered. There are many web accessibility tools provided by the W3C used to assist developers in providing an accessible website. In order to encourage web developers to consider user accessibility when developing their websites, the $\mathrm{W} 3 \mathrm{C}$ created a narrative set of guidelines, the Web Content Accessibility Guidelines (WCAG). Performing accessibility and evaluation checks of 
the technical parameters of a website can be used to identify possible barriers to users with visual impairments. When the the errors have been identified, amendments can be performed then the errors can be rectified and it is then possible to vastly improve the performance of the website.

\section{B. Testing Tools}

The testing tools used in this research work determine the conformance of the e-commerce websites against accessibility checks and also determine the conformance to the current accessibility guidelines, mainly the WCAG 2.0 (AA). A select few of the testing tools will also investigate and evaluate web accessibility by users with colour blindness.

\section{Automated testing}

Automated testing tools are an effective method of highlighting potential errors and usability issues. Predominantly the automated testing tools are performed by online validators such as $\mathrm{W} 3 \mathrm{C}$ validation service and automatically detect issues such as missing alternative text attributes and undefined document language types. However, manual intervention is required in order to correct the problems highlighted. The tools used to perform the audit are specified below:

- W3C Mark-up Validation Service (http://validator.w3.org/). The W3C validation service is an online testing tool that checks the validity of web documents in HTML, XHTML etc.

- Colour Blind Webpage Filter (http://colorfilter.wickline.org/). The Colour Blind Webpage filter is a simulation tool that reveals how a website looks to a user with colour blindness, including examples of the conditions; Protanopia, Deuteranopia, Tritanopia, Achromatopsia etc. simulating how the snapshot is viewed by a person with a colour blindness condition.

- Etre Web Accessibility Tool (http://www.etre.com/tools/accessibilitycheck/). The Etre Web Accessibility Tool evaluates the webpage against the WAI guidelines, relating to accessibility.

- Achecker Web Accessibility Checker (http://achecker.ca/checker/index.php). The Achecker Web Accessibility checker checks single HTML pages against a range of accessibility standards, including the WCAG 1.0 \& 2.0 .

\section{Audit Reporting Structure}

First and foremost the authors will conduct a validation check on the HTML and CSS Mark-up language of the chosen website Amazon.co.uk. A validation check is a test to ensure that the Mark-up used conforms to the defined language type. The identified automated tool for checking compliancy and Mark-up errors is the W3C Mark-up Validation Service tool. The audit structure will be conducted by using each of the tools listed above to test each of the consecutive pages of the website. The homepage, search related pages, product/catalog pages and lastly the checkout pages will all be tested individually using the tools identified. The tools will reveal the current usability issues and compliancy failures of the current major online retail businesses and provide detailed reports on the errors users with disabilities may face. This audit is focusing on the barriers faced by users with sight deficiencies such as colour blindness. The methodologies will enable the gathering of required improvements and barriers to overcome to produce a framework for an accessible e-commerce website.

\section{E. Audit of Amazon.co.uk}

Users form their first impressions of a website homepage almost instantaneously, perceiving the colour scheme, credibility and usability of the website. Whether or not a user will purchase from the site is influenced by the credibility of the webpage i.e. whether or not it looks professional or amateur. To identify issues of Mark-up and CSS, the Amazon.co.uk homepage (www.amazon.co.uk) was entered into the address field of the W3C Mark-up Validation Service. Both the character encoding and document type was set to 'detect automatically' to see whether or not the document and coding types had been identified within the code.

In Amazon website, before the validation output report, the check identified the document type (DOCTYPE) was not stated within the document. Therefore, the validation report was forced to roll-back and validate the page against the default doctype; HTML 4.01 Transitional. Many errors were identified in the findings of the validation report; this is due to the undeclared doctype which forces the validator to validate using a different doctype, which is more than likely to be the incorrect and generate false error messages. Before the Amazon.co.uk page can be declared as valid, a valid doctype is required. Having performed the general W3C validation service report, a more visual user-focused tool was used to identify the potential errors of the website homepage. A web accessibility check was performed using the "Etre Web Accessibility Tool". The tool compares the submitted URL against the WAI accessibility guidelines and evaluates the findings. According to the Etre Web Accessibility test results, there is one critical error that needs to be fixed for the website to comply with the World Wide Web Consortium (W3C)'s Web Accessibility Initiative (WAI) guidelines. As it is a priority 1 requirement, the error must be fixed in order to provide the minimum, most basic level of accessibility. 35 other problems were identified and should be fixed providing the minimum accepted level of accessibility recommended by the European Union (EU). A 'may fix' error is suggested to be fixed to increase and maximize accessibility. The testing report indicated that one of the problems discovered was that nested tables were used i.e. tables placed within tables. Screen readers read website content in a liner fashion, however nested tables linearise poorly, therefore; the process by which a screen reader or speech browser interprets the content is compromised. Screen readers interpret html table's cell by cell (left to right, top to bottom) which is the correct sequence for reading tabular data. However, nested tables do not work well with this process. One of the other errors listed within the report is the use of <iframe> elements with missing longdesc attributes. Often, a title attribute alone is not enough to explain the iframe content; therefore it is recommended that a long description attribute is used to further explain the content and importance to blind users. The use of a long description attempts to convince blind users that accessing the content is worth their while. However, the longdesc attribute is poorly supported by the leading web browsers, therefore in order to provide the user with a description, a simple link to a page that describes the iframe content is required. Solely the title tag alone is not enough to inform a blind user of the 
content within the frame. Therefore, the website does not conform to the WAI guidelines, specifically guideline; H45: Using Longdesc. When an absolute font size is used on the web site, users who have a visual impairment are unable to resize the font as the font is static (often an image or text as opposed to using HTML and CSS styling). In terms of ecommerce and visually impaired users, if users are unable to resize text, users are unlikely to read the details of products, which in turn make it unlikely that the user will purchase a product. This would result in a potential loss of sale through a denial of service to users with disabilities. Having highlighted numerous accessibility problems using the tools above, it was then decided that a more in depth, detailed approach should be used to discover the actual errors of the homepage, with reference to specific guidelines of the WCAG 2.0 and the specific lines of code where the error exists. The web accessibility checker by Achecker provides a more detailed reporting structure highlighting the conformance errors (to WCAG 2.0), specific errors within the code and identifies what needs to be amended to remove the accessibility barrier. The report format was structured to view the errors by their corresponding guideline. The guidelines to check against was selected as; WCAG 2.0 (Level AA) - A web developer should conform to this checkpoint, checkpoints 1 and 2. The check results highlighted 11 known problems, 92 potential problems, 251 HTML validation problems and lastly 9 CSS validation problems. The majority of errors reported were related to the insufficient contrast ratio of the foreground and background colour of the active link text. The repair note suggested that a sufficient contrast of 4.5:1 for standard text, or 3:1 for larger text was required in order to comply with guideline 1.4.

"1.4 Distinguishable: Make it easier for users to see and hear content including separating foreground from background.

Success Criteria 1.4.3 Contrast (Minimum) (AA).

Check 303:The contrast between the colour of active link text and its background is not sufficient to meet WCAG2.0 Level AA.”[5].

The report signified that Amazon.co.uk failed to include an alternative text attribute for an image used as an anchor, i.e. the purpose of the image is not stated. If the image is used as a link an alt attribute is required to describe the link destination. As discovered in the early analysis of the homepage using the $\mathrm{W} 3 \mathrm{C}$ validation service tool, the document doctype is missing, conflicting with guideline 3.1 Readable: Make text content readable and understandable; Success Criteria 3.1.1 Language of Page (A). A HTML element is required to have a "lang attribute" as the attribute allows assistive technologies such as screen reader software to adapt and pronounce the syntax that matches the language of the content. Amazon.co.uk has failed to state a language type on the opening HTML element. To repair the missing doctype element, Amazon.co.uk should implement the following:

<html xmlns="http://www.w3.org/1999/xhtml" $\mathrm{xml}:$ lang="en-gb" lang="en-gb">

The colour blind webpage filter is a simulator that enables a user with full vision to reveal how an image may appear to a user with a colour blindness condition. The filter allows the user to select a colour filter and simulates how the website would be viewed by someone with a colour blindness condition. The tool simulates a variety of colour blindness conditions; Protanopia, Deuteranopia, Tritanopia, Monochromacy. According to the result of Protanopia test, the appearance of the Amazon website appears largely the same, albeit a little contrast reduced. However the links, menu and general use of colour is appropriate for users with Protanopia. Deuteranopia filter test results appeared largely the same as the results for Protanopia. The results can be explained by the conditions; Protanopia - no red cones and Deuteranopia - no green cones. As the website homepage doesn't feature these colours the webpage remains unaffected and is viewed similarly, if not the same as how a user without a colour blindness condition views the webpage. Users with Tritanopia colour blindness condition (blue/yellow colour blindness) have missing blue cones, therefore it is expected that the screenshot will appear different in colour. Cases of Tritanopia are very rare, but users who have the condition are sensitive to the colour blue. There is a significant change in colour of the original 'orange' elements of the website as it appears a shade of pink. The original light blue is now a darker, high contrast blue, which could potentially throw up errors when roll-overs are used on active menu links. Lastly, when the Monochromacy filter was applied, the website was in greyscale, only varying in contrast and brightness. In terms of visibility for colour blind users, the Amazon website performance and overall user experience is excellent as the webpage renders legibly. Amazon.co.uk has chosen colours that contrast well and appears largely the same for most colour blind users.

\section{a) 3.4.2. Search pages}

A search facility is a critical, necessary function of any ecommerce website. In order for a user to purchase a product, they need to be able to locate it. The search must have a simple user interface and search the entire website and the results must be legible to all users. "Site designers must create sophisticated, but simple search engines capable of delivering the goods on the user's first search query." [6]. Manual search was conducted using the main search feature of the Amazon.co.uk website. The results of key words used are listed in a structured manner in Amazon web site, categorized by the relevance of the product to the search term. The results display a thumbnail image of the product and are upfront with the total cost of the item including shipping. Red and green text is used to highlight delivery times and pricing, which could potentially cause problems for users with Protanopia and Deuteranopia. The colour blind simulator was used to check for the potential issues.

\section{b) 3.4.3. Product page}

An Accessible product page is a necessity. If users are unable to use the product page, items cannot be purchased. Using the Achecker Web Accessibility Checker to check for errors against the WCAG 2.0 (AA), numerous avoidable errors were reported, including missing alternative text and colour contrasting issues. Again, the issue of the undeclared doctype impeded the results. The audit report identified 11 know problems, all of which could be rectified with little effort to ensure full accessibility by visually impaired users. The product details, such as the dimensions and page statistics appear midway down the product page. The description along with the product details should have placement towards the top of the product page as they have more relevance to the product and are of more importance to the user than what other users have previously bought. The customer reviews elongate the height of the page, making it an arduous task to scroll to the bottom of the webpage. The customer reviews do not have a character limit and display the full review elongating the page unnecessarily. A line break, such as '... read more' should be 
implemented, allowing the users to click to read more if they wish to do so. A character limit should be introduced to constrain the length of the 3.4.4. Checkout page. For the purpose of this audit, an item was added to the basket and checkout process proceeded. It was apparent that Amazon.co.uk requires users to register with the website before they are able to checkout with the product. Requiring users to register first as opposed to checking out as a 'Guest' puts off users from using the website, as they may wish to use the website as a one off and do not want marketing emails associated with the account. The previous checkout stages were arduous and took a considerable amount of time to complete. Amazon has separated the checkout process into several stages to make the process easy to complete, however there are too many stages. A total of 7 stages of entering information were required before the product order could be placed. Amazon.co.uk could do with reviewing, simplifying and streamlining their checkout process in order to achieve a more usable user-interactive checkout.

\section{E-COMMERCE GUIDELINES}

In this section the authors will propose a set of e-commerce specific guidelines derived from the results of the early analysis of the major e-commerce websites in order to identify the usability issues faced by users with a visual impairment. The following framework guidelines aim not to replace, but to comply to and be applicable to all e-commerce websites.

\section{A. General}

\section{1) Guideline 1.2}

It is important to make good use of headings, sub headings and you can use breadcrumb navigation to inform users of their position on the website. Text headings and sub headings that are analysed by search engines are headings such as $\mathrm{H} 1$, $\mathrm{H} 2$, and subheading H3. Paragraphs are also analysed for readability, including the alignment, font used and numeric value of the words. Most users scan text rather than read website content, therefore it is a good idea to provide users with a heading hierarchy and use sub headings where possible to outline to the user what the content is about. Web Credible (2005) recommend that developers should be aiming for one sub-heading every two to four paragraphs. Most importantly, the sub-headings should group on-page content into logical groups, to allow site visitors to easily access the information that they are after [7]. A virtual breadcrumb trail can be created to help users navigate within websites and provide users with the information as to where the user is within the site, allowing the user to 'jump' to any previous page in the trail without having to use the browser navigation tools [8]. Thus, usability can be enchased using this method more effectively. Rogers and Chaparro et al (2012) indicated that; of the participants that were exposed to a site with a breadcrumb trail $(n=30), 40 \%$ used the breadcrumb five or more times to navigate on the site (Range $=5-31, n=14$ ). However, this accounted for only $6 \%$ of the navigation overall. The Back button, the main Navigation bar, and embedded links were used the majority of the time [9].

\section{2) Guideline 1.2}

A text equivalent for every non-text element should be provided (e.g. via 'alt', 'longdesc', or in element content) [10]. It is essential for a developer to include alternative text attributes on non-text elements such as images as visually impaired users have difficulty viewing images and other media rich content, therefore an alternative description is required. This includes: images, graphical representations of text (including symbols), image map regions, animations (e.g., animated GIFs), applets and programmatic objects, ASCII art, frames, scripts, images used as list bullets, spacers, graphical buttons, sounds (played with or without user interaction), stand-alone audio files, audio tracks of video, and video [11].

\section{3) Guideline 1.3}

Use Mark-up and CSS where possible allowing text-size \& other accessibility functions to operate correctly. The use of mark-up allows developers to consistently provide information to the viewer dynamically as using mark-up improperly hinders accessibility [12]. The use of HTML 'lang' attribute within the header of the HTML page defines the language used. Defining the language attribute assists screen reader speech synthesisers in rendering the content in a more direct and meaningful manner, based upon the language chosen and the cultural practices of the chosen language. E.g. defining the HTML language as 'en' defines the language as English which is culturally read in a linear manner from left to right and 'en-gb' contains a regional sub tag and defines the language as British English, taking into account the distinction between counties that predominantly use the English language. The use of correctly written mark-up is essential for web-based screen reader software. As an example of the latter, typically blind or severely vision impaired users will use synthesised text-tospeech (TTS) software in order to access websites and interpret website content. However, improper hierarchical use of headings such as $\mathrm{H} 1, \mathrm{H} 2$ and $\mathrm{H} 3$ sub-headings can confuse screen reader software, often resulting in a parse error; 'there are no headings on this webpage'. If a developer uses an image of a title rather than CSS styling the title, the font increase and decrease functions will not work as the title is static, therefore incomprehensible by users with low vision. If alternative text attributes for graphical images are provided, screen readers will pick up and explain the relevance of the graphic to the user, however, if missing; the user could miss out on useful or significant information. "Screen readers can only read textual elements of web pages, so graphics and scripts cannot be interpreted" [13]. It is essential that developers control the website modules using CSS styling as apposed using images to convey the information. If graphical images and/or animations are used, relevant alternative text attributes must be provided in order to explain the importance of the image. The alternative text should also describe the appearance of the visual content of the image, to convey the illustration. Users with sight impairments often view small portions of the website content at one given time and may also use screen reader software to convey the content of the webpage, therefore the content is required to contain sufficient headings, tab index, tables, structured navigation etc. in order to serve its purpose and portray the content to the user. The Web Content Accessibility Guidelines 1.0 (WCAG) states that "when an appropriate mark-up language exists, use mark-up rather than images to convey information.[Priority2]" [14].

\section{4) Guideline 1.4}

Website logo should be placed to the left and linked to the website homepage. Linking the website logo to the website home page assists users in navigating home. The aim is to provide as many routes possible to the website pages through the use of strategically placed links, enhancing the usability of the website. The logo area needs to be prominent and attract the attention of the user when the user enters the website. The 
upper-left corner is usually the best placement for languages that read from left to right [15]. A tag line of less than 100 characters may also be placed below the logo to summarise the website content. However, a tag line may not be required if the company logo is self-explanatory.

\section{5) Guideline 1.5}

Ensure there is sufficient contrast between the background and foreground ensuring visibility is consistent for all users. To accommodate colour deficient users, such as users with Monochromacy a sufficient high colour contrast option must be used. Text and background colours must be legible and of a sufficient colour contrast. Select colour combinations that contrast well together and increase lightness between colours. Make use of colour hexadecimal and/or RGB numbers over names of colours in the CSS style sheet. In accordance with the WCAG 2.0 guidelines a 1.4.3 Contrast (Minimum) should be used. The visual presentation of text and images of text has a contrast ratio of at least 4.5:1, except for the following: (Level AA) Large Text: Large-scale text and images of large-scale text have a contrast ratio of at least 3:1; Incidental: Text or images of text that are part of an inactive user interface component, that are pure decoration, that are not visible to anyone, or that are part of a picture that contains significant other visual content, have no contrast requirement. Logotypes: Text that is part of a logo or brand name has no minimum contrast requirement. [16].

\section{6) Guideline 1.6}

Provide the option of resizing text; increase and decrease text-size. An option to resize text is an essential aspect of web design to enable users with disabilities to operate the web. Most commonly, web browsers have the ability to change the text size of web pages however, if static text is used the text is unable to be resized. Developers cannot solely rely on the user's web browser to alter the text size. An accessibility option to resize text shows the user that the website is user friendly and is accessible by users with a visual impairment. A user with a visual impairment may be required to increase the text size to be able to read the content as they may struggle to read small text. Therefore, the option of increasing the text size enables the user to read the content more easily, thus removing the barrier of illegible content. The text size is required to be increased up to twice the original text size i.e. 200\%. For many users with disabilities such as visual implications, the use of a keyboard to navigate a website is required. Aim to ensure that all website functionality can be performed by the use of the keyboard only, without including timing requirements for specific keystrokes.

\section{B. Navigation}

1) Guideline 2.1 "Design consistent navigation that is simple, intuitive and obvious and remain in the same locations on each page". Design pages to use consistent navigation methods. This is particularly important for people with low vision using screen magnifiers. Since the users with low vision cannot see the entire screen at once, the more they can predict where elements are, the easier it is for them to get an overall feel for a page. It is important that navigation controls must be located in the same location on every page. The navigation title attributes must also be self-descriptive matching the headings of the destination pages. Child menus should only be used if necessary and the links should be direct to the relevant placement of the pages.

2) Guidelines 2.2

Navigation must make use of roll-over and highlighting functions providing feedback to the user of their position on the navigation. Orientation cues are excellent for providing the user with feedback as to where they have placed their cursor and also the highlighted navigation will indicate what page the user is on. Wayfinding orientation will inform the user of their current position within the website.

\section{Search Functions}

\section{1) Guideline 3.1}

A search function should be visible and remain on every page in the same position. According to Proctor et al (2005) the search function is the most important feature of an e-commerce website. Therefore, it is recommended that the search function be placed in a position users expect to find it, like the upper-right or upper-left corner of a page. For users with low vision or no vision, this is particularly important. The search feature must be in the same position on each and every page and be of adequate width with enough space for at least 30 characters.

\section{2) Guideline 3.2}

Advanced search and filter options should be considered to refine the search results. An advanced search feature is a necessary feature of any e-commerce website. The advanced search allows users to refine the results that they seek, enhancing the usability of the website. It is recommended that the user is able to perform a search of specific categories to assist in discovering the search results. To ensure that the search is kept as simple as possible offer users the option to do an advanced search only when the search results are presented.

\section{3) Guideline 3.3}

The search function must allow users to search by product name, SKU code, category, manufacturer and product price. The search feature must have the ability to search the entire website. The search function should also allow users to search by product name, SKU code, category, manufacturer and product price in order to help users refine their search category and results.

\section{Product Catalog}

\section{1) Guideline 4.1}

Product layout must be consistent and in a grid format e.g. three products per row. Products must be categorised and in a layout that is clear, concise and correctly formatted. The product layout should remain uncluttered and make use of whitespace. Dependant on the amount of space, it is recommended that you use three products per row with sufficient padding between product images to help individualise products.

\section{2) Guidelines 4.2}

Present accurate, consistent and detailed descriptions and images of products[17]. Product descriptions should remain accurate, descriptive and provide the user with sufficient information about the product. The product page should present the details of a single product only to avoid confusion between other products; however related products may be 
displayed as a secondary focus towards the bottom of the product page. The product image should be recognisable, accurate and of high quality with as few visual distractions within the image. "Recognising a familiar item visually is easier for some people than recalling its name." (Nielsen et al, 2000). The users should be provided with clickable thumbnail images providing several views of the product from all angles, including different variations of the product such as product colours. However, the importance of displaying multiple images depends entirely on the product. If a detailed image is not required, then it is not necessary to provide the user with one. According to Nielsen et al (2000) the users sometimes wanted to see other views of items (especially clothing and furniture) [18]. Nielsen (2000) further adds that to accommodate users who have difficulty seeing, or who turn off images on their browsers, it is important to provide meaningful ALT text. ALT text is what appears when the user has turned off images, and it is also what screen readers, or voice browsers, speak [19].

\section{3) Guideline 4.3}

Provide users with the total product price (and currency); be upfront with pricing on both the category and product pages. One of the main factors of success of an E-commerce website is that of honesty. An honest retailer will gain the trust of the user i.e. the person's willingness to invest time, money, and personal data in an e-commerce site in return for goods and services that meet certain expectations [20].

\section{4) Guideline 4.4}

Provide sufficient product availability information. Products should remain on the product page even if out of stock. Availability information is important to users. If a product is out of stock and if this is not indicated on the website, allowing a user to purchase the product it would take a longer amount of time to be delivered, thus sacrificing the buyers trust. The length of time that the user is willing to wait can depend on several factors, including how much the user wants the item and whether the user can obtain a suitable alternative more quickly. If a product is not available right away, the user may opt to:

- Wait for the site to ship the item

- Shop elsewhere

- Give up on that item, perhaps choosing something else

- Buy nothing [21].

5) Guideline 4.5

Users should be able to add items to their shopping cart without having to register first. Nielsen et al (2000) states that the registration process on web sites must always be optional. Requiring registration before or during a purchase drives away business. If a user fails or refuses to register, and registration is required, the failure results in a sales catastrophe for the site, which is not what either the prospective buyer or seller intended. People who give up on a website may not be back again [22]. Allowing customers to register optionally after a purchase is much better, because then it does not interrupt or prevent the sale, and it is less offensive to customers because they have a choice [23].

\section{E. Shopping Cart and Checkout}

\section{1) Guideline 5.1}

Simplify and streamline the whole ordering process, obtain only required information. It is important to simplify the ordering process of purchasing a product to allow users to checkout with their desired shopping basket as smoothly and as quickly as possible. By simplifying the process, obtaining only required information; users are less likely to abandon their shopping cart if they are faced with an abundance of required text fields irrelevant to the purchasing of the product.

\section{2) Guideline 5.2}

Show users the checkout stages using a progress bar and highlight where they are upon checkout. Users should be provided with a linear procedure to completing the checkout process. A progress bar identifies at what stage the user is at upon checkout. An indication of the steps to complete is often enough to encourage shoppers to checkout and purchase the items in their shopping cart. Keeping the user informed of their progress at all times puts the user at ease. Illustrations of the checkout processes are often used as an indication of progress, having the steps to complete 'greyed out' as a secondary step to the current step to be taken. However, the Nielsen Norman Group (2000) discovered that upon checkout; "the only problem with these graphics was that users sometimes tried to click the graphic to skip to a later step, typically shipping, because they had questions about that step of the process" [24]. To address this problem, the user should be provided with tool tips and guidance on how to checkout, providing obvious action buttons to continue to the next checkout step.

\section{3) Guideline 5.3}

Highlight required form fields. When designing and developing form fields it is wise to use a CSS Focus state to help specify which form field the user has clicked on, highlighting the field ready for user input. The CSS example below portrays the use of a border colour to signify the form field selected. It is also important to highlight incorrect or missing required form fields that the user may have missed. Required fields are often marked by an asterix (*). This is often standard procedure on many websites upon checkout and registration, however if a required field is missed such as a credit card expiry date then the required fields become highlighted upon validation. A red border colour is often used to highlight a missing required field as the colour red is associated with an error or wrong doing.

\section{4) Guideline 5.4}

The shopping basket is to be placed in the upper most top right hand position of the E-commerce website and indicate if it contains products. The shopping cart must explicitly display the active content within the cart enabling the user to retrieve and checkout their chosen products at a later stage. When a user adds an item to their shopping cart, provide a link to checkout and alternatively provide the user with a link to 'continue shopping'. According to P. Markellou et al (2005) the best location to place the shopping cart is at the right top area as users will look there first to find the link. A visual indication whether the cart contains product must be shown in the shopping cart. [25]. Typically when an item is added to the cart, the e-commerce site will create and store an HTML 'cookie' either on the client's browser or server side; if the client has selected an option to prevent cookies from being stored on their machine. Users with visual impairments often memorise and/or visualise website framework to allow them to operate the website with ease.

\section{5) Guideline 5.5}

Highlight delivery costs on order summary before the purchase is made. 
If a product demands a fee for delivery, the cost must be clear in the early stages of the checkout process. The number one cause of abandoned shopping carts is the shipping pricing is too costly and is only revealed upon the final checkout stages.

\section{6) Guideline 5.6}

Display help and tool tips for form fields. If text fields are unobvious, provide the user with tool tips in order to explain the details required. The tool tips are often displayed if a user places the mouse cursor over the title of the required field as a directional tip to how to perform the procedure or complete the required form field. Tool tips may also appear as validation is put into place, such as a missing required form field. A tool tip will be displayed explaining the reasons for the submission error.

\section{CONCLUSION}

This research study and the development of the framework have covered the aspects of the usability issues faced by users with visual impairments and have provided a solution as well as recommendations for accessible user interface design. In addition, the research study has provided an example of measures to improve accessibility for the visually impaired person. The audit has helped to uncover the unique requirements of those who have a visual impairment and has provided developers with a framework to assist them in producing usable website designs. The testing methods used within this paper have proven to be successful; a select few tools were visual specific, such as the colour blind tool. The testing outcomes of the proposed prototype identified that the measures for designing an accessible user interface were met, complying with current guidelines as proposed by the $\mathrm{W} 3 \mathrm{C}$ and by that of the identified guidelines of the framework. During the implementation of the framework, the guidelines were used as a reference to accessible user interface design. The prototype website has made use of modern technologies, (including CSS3 elements) and design techniques without compromising the accessibility of the visually impaired. This research study has provided developers with new standards to follow in developing e-commerce websites that are accessible for users with a visual impairment. The framework has proven to eliminate the errors outlined in the initial audit and make ecommerce websites more usable, catering to the requirements of the visually impaired. For future work the development will focus on implementing the framework to current noncompliant e-commerce websites and the integration of web browsers, such as Google App browser plugins that specifically address the errors, including the integration of assistive technologies, mobile devices and mobile applications implementing the framework into all web-browser devices.
[4] Equality Act 2010. Legislation.gov.uk. [Online] 2010. [Cited: 15 February,2012.] http://www.legislation.gov.uk/ukpga/2010/15/part/3/crossheading/provis ion-of-services.

[5] Web Content Accessibility Guidelines (WCAG) 2.0. W3C - WCAG 2.0. [Online] [Cited: 19 December 11.] http://www.w3.org/TR/WCAG/.

[6] Nielsen J, Rolf Molich, Carolyn Snyder, Susan Farrell. E-commerce user Experience - Search. s.1. : Nielson Norman Group, 2000.

[7] Webcredible. Web Usability Guide. London, 2007.

[8] Levene, Mark. An Introduction to Search Engines and Web Navigation Vol. Second Edition. ISBN 978-0-470-52684-2.., 2010

[9] Bonnie Lida Rogers and Barbara Chaparro. Breadcrumb Navigation: Further Investigation of Usage. Psychology.wichita.edu. [Online] [Cited: $27 \quad$ February 2012.] http://psychology.wichita.edu/surl/usabilitynews/52/breadcrumb.htm.

[10] Frank, Jonathan, Web Accessibility for the Blind: Corporate Social Responsibility or Litigation pg. 8. Sawyer Business School, Boston : s.n., 2008.

[11] Web Content Accessibility Guidelines 1.0 - Alt text. W3C. [Online] 06 November 2000. [Cited: 12. 27 February 12.] http://www.w3.org/TR/WCAG10-HTML-TECHS/\#image-textequivalent.

[12] Web Content Accessibility Guidelines - Markup. W3C. [Online] 05 May 1999. [Cited: 27 February 12.] http://www.w3.org/TR/WCAG10/\#g1structure-presentation.

[13] Alan Dix, Janet Finlay, Gregory D. Abowd, Russell Beale. HumanComputer Interaction Third Edition. s.1. : Pearson Education Ltd, 0-13046109-1., 2004.

[14] Web Content Accessibility Guidelines 1.0 - Techniques for use of Markup. W3.org. [Online] 06 November 2000. [Cited: 21 February 2012.] http://www.w3.org/TR/WCAG10-TECHS/\#tech-use-markup.

[15] Web Content Accessibility Guidelines 1.0 - Techniques for use of Markup. W3.org. [Online] 06 November 2000. [Cited: 21 February 2012.] http://www.w3.org/TR/WCAG10-TECHS/\#tech-use-markup.

[16] Robert W. Proctor, Kim-Phuong L. Vu. Handbook of Human Factors in Web design pp. 514-527. s.1. : Lawrence Erlbaum Associates, Inc.,. ISBN 0-8058-4612-3., 2005.

[17] Nielsen, Kara Pernice and Jakob Nielsen . Beyond ALT Text: Making the Web Easy to Use for Users pp. 80. Fremont, California : Nielsen Norman Group, 2001.

[18] Nielsen, Kara Pernice and Jakob Nielsen. Beyond ALT Text: Making the Web Easy to Use for Users with Disabilities pp. 56 Fremont, California : s.n., 2001.

[19] Noorfadzilah Md Zainudin, Wan Fatimah Wan Ahmad and Goh Kim Nee. Designing E-commerce User Interface Sri Iskandar, Perak, Malaysia : s.n., 2010.

[20] Jakob Nielsen, Carolyn Snyder, Rolf Molich, Susan Farrell. ECommerce User Experience - Product Pages pp. 18 - . s.l. : Nielsen Norman Group, 2000.

[21] Jakob Nielsen, Rolf Molich, Carolyn Snyder, Susan Farrell. E-commerce User Experience - Trust pg. 2. s.1. : Nielsen Norman Group, 2000.

[22] Jakob Nielsen, Susan Farrell, Carolyn Snyder, Rolf Molich. E-commerce User Experience - Checkout \& Registration pp. 2 -. s.l. : Nielsen Norman Group, 2000.

[23] P. Markellou, et al. Web Systems Design And Online Behaviour Product Catalog and Shopping Cart Effective Design pp 232-250. s.1. : Idea Group Publishing, 2005.

\section{REFERENCES}

[1] V. Zwass "Electronic Commerce: Structures and Issues pp. 3 - 23. Vol. 1. 1996.

[2] Schneider, Gary P. Electronic Commerce. San Diego, Course Technology, 9th ed,. 0538469242. 2010.

[3] Web Loyalty - News; Online Retail to Exceed £37 Billion by 2014. Webloyalty. [Online] Webloyalty, 18 April 2011. [Cited: 06 March 2012.] http://www.webloyalty.co.uk/news-a-research/30-press-releases2011/80-online-retail-to-exceed-p37-billion-by-2014. 